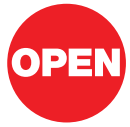

SUBJECT AREAS: APPLIED PHYSICS

MECHANICAL PROPERTIES

NANOSCALE MATERIALS

METALS AND ALLOYS

Received

21 July 2012

Accepted

1 October 2012

Published

25 October 2012

Correspondence and requests for materials should be addressed to

H.Y.

(yuhailiang1980@ tom.com; hailiang@ uow.edu.au)

\section{Asymmetric cryorolling for fabrication of nanostructural aluminum sheets}

\author{
Hailiang YU',2, Cheng LU', Kiet TIEU', Xianghua LIU³, Yong SUN', Qingbo YU³ \& Charlie KONG ${ }^{4}$
}

${ }^{1}$ School of Mechanical, Materials \& Mechatronic Engineering, University of Wollongong, NSW 2500, Australia, ${ }^{2}$ School of Mechanical Engineering, Shenyang University, Shenyang 110044 , China, ${ }^{3}$ State Key Laboratory of Rolling and Automation, Northeastern University, Shenyang 1 10004, China, ${ }^{4}$ Electron Microscope Unit, University of New South Wales, Sydney, NSW 2250, Australia.

Nanostructural Al 1050 sheets were produced using a novel method of asymmetric cryorolling under ratios of upper and down rolling velocities (RUDV) of 1.1, 1.2, 1.3, and 1.4. Sheets were rolled to about $0.17 \mathrm{~mm}$ from $1.5 \mathrm{~mm}$. Both the strength and ductility of Al 1050 sheets increase with RUDVs. Tensile strength of Al sheets with the RUDV 1.4 is larger $22.3 \%$ of that for RUDV 1.1, which is $196 \mathrm{MPa}$. The TEM observations show the grain size is $360 \mathrm{~nm}$ when the RUDV is 1.1 , and $211 \mathrm{~nm}$ for RUDV 1.4 .

$\mathrm{n}$ recent years, a lot of interest has been shown in the production of materials with nano-sized grains, especially in bulk through Severe Plastic Deformation (SPD) techniques. This interest is due to unique physical and mechanical properties inherent to various nanostructural materials. The SPD techniques, which include highpressure torsion $^{1}$, reciprocal extrusion ${ }^{2}$, equal-channel angular pressing (ECAP) ${ }^{3}$, accumulative roll bonding ${ }^{4,5}$, repetitive corrugation and straightening ${ }^{6}$, constrained groove pressing ${ }^{7}$, equal channel rolling ${ }^{8}$, asymmetric rolling, cryorolling, etc, have been developed to fabricate bulk nanostructural or ultrafine grain samples of different metals. Compared with above SPD techniques, the asymmetric rolling and cryorolling employed have potential for large-scale industrial applications of nanostructural materials.

In asymmetric rolling, sheets are rolled between rolls that either are of different diameters, or are rotating at different velocities. Asymmetric rolling has a potential for industrial applications because it involves a decrease in the rolling pressure and torque and an improvement of the rolled strip shape ${ }^{9,10}$. In addition, it is generally claimed that during asymmetric rolling, the complete strain state imposed on the strip is a combination of plane strain deformation and of an additional shear component imposed on the rolling plane in the rolling direction ${ }^{11,12}$. Several studies have shown that this additional shear strain contributes to grain rotation and subdivision producing grain refinement and modification of crystallographic texture of the material that can improve the properties of the sheet during subsequent plastic deformation process ${ }^{13,14}$. Al alloy strips can exhibit high formability if they are produced via the shear deformation ${ }^{15}$. The possibility of generating shear textures across the whole volume of the strip if the shear deformation associated with rolling is strong enough to deliver the shear strain to the centre of the strip. The asymmetric rolled strip exhibited uniform microstructures across the thickness direction, in contrast to the conventionally rolled strip, and a strength comparable to or exceeding that of the commercial $\mathrm{Al}$ alloy strip commonly in use.

Cryorolling is a simple low-temperature processing route that requires a relatively lower load to induce severe strain for producing the sub-microcrystalline structural features in materials. The method using rolling under liquid nitrogen temperature has been widely used to improve the materials properties ${ }^{16-22}$. The cryorolling may be easily adapted for large-scale industrial applications of nanostructured materials ${ }^{16}$. Wang et al ${ }^{17}$ described a thermomechanical treatment of $\mathrm{Cu}$ that results in a bimodal grain size distribution, with micro meter-sized grains embedded inside a matrix of nanocrystalline and ultrafine grains. The matrix grains impart high strength, as expected from an extrapolation of the Hall-Petch relationship. Meanwhile, the inhomogeneous microstructure induces strain hardening mechanisms that stabilize the tensile deformation, leading to a high tensile ductility elongation to failure, and 30\% uniform elongation. Cryorolling has been identified as one of the potential routes to produce bulk ultrafine grained $\mathrm{Al}$ alloys from its bulk alloys ${ }^{18}$. The microstructure and mechanical properties of a precipitation hardening $\mathrm{Al}-\mathrm{Cu}$ alloy subjected to cryorolling, low temperature annealing and ageing treatments were studied by Rangaraju et $\mathrm{al}^{20}$. Under optimal processing conditions, ultrafine grained microstructure with improved tensile strength and good ductility was obtained. Due to the suppression of dynamic recovery during cryorolling both the tensile strength and yield strength were considerably increased. Moreover, the cryorolling 
process offers other advantages, such as, lower required plastic deformations, simple processing procedures and ability to produce continuously long length product, as compared to other severe plastic deformation processes ${ }^{21}$.

Recent developments in the field of both asymmetric rolling and cryorolling processes have led to a renewed interest in improvement of the grain refinement of materials. However, no research has been found that surveyed materials using the methods together. In this paper, the nanostructural $\mathrm{Al} 1050$ sheet was produced by asymmetric cryorolling technique. Meanwhile, the mechanical properties of $\mathrm{Al}$ 1050 under different ratios upper and down rolling velocities (RUDV) were studied. When the RUDVs increase from 1.1 to 1.4, both the strength and the ductility of Al 1050 sheets increase.

\section{Results}

Fig. 1 shows the mechanical properties of the rolled Al 1050 sheet. In Fig. 1(b), with increasing the RUDVs, both the yield stresses and tensile stresses of the sheets increase. The tensile stress is $160 \mathrm{MPa}$ when the RUDV is 1.1, which reaches $196 \mathrm{MPa}$ for RUDV 1.4 with an increase of $22.3 \%$. Meanwhile, with increasing strength, the ductile also increases slightly as shown in Fig. 1 (c).

Fig. 2 shows the TEM graph of the samples after asymmetric cryorolling process. Compared Fig. 2 (b) and (c), the grain size in Al 1050 sheet with the RUDV 1.1 is much larger than that with the RUDV 1.4. After rolling process, the grain size is $360 \mathrm{~nm}$ when the RUDV is 1.1, and the grain size is $211 \mathrm{~nm}$ when the RUDV is 1.4.

Eq. (1) shows the Hall-Petch relationship,

$$
\sigma_{r}=\sigma_{i}+k_{y} D^{-1 / 2}
$$

where $k_{y}$ is Petch parameter, $D$ is grain size. Sato et al $^{23}$ analyzed the Hall-Petch relationship of Al 1050 in friction stir welds of equal channel samples. The extrapolated value for a boundary-free condition and slope of the Hall-Petch equation give values of $H_{0} \approx 18 \mathrm{Hv}$,

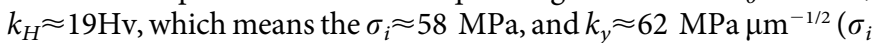
and $k_{y}$ equal $3 \sim 3.5$ times of $H_{0}$ and $k_{H}$ respectively). According to Eq (1), the calculated tensile stress for RUDV of 1.1 is $161 \mathrm{MPa}$, and that for RUDV of 1.4 is $193 \mathrm{MPa}$. They are in agreement with the measured values.

\section{Discussion}

The suppression of dynamic recovery during deformation at extremely low temperatures is expected to preserve a high density of defects generated by deformation ${ }^{24}$. With decreasing the deformation temperature, the strength of Aluminum alloys generally increases. Moreno-Valle et $\mathrm{al}^{25}$ studied the strength properties of an $\mathrm{Al} 6061$ alloy at room and cryogenic temperatures using high pressure torsion. A decrease of the testing temperature results in improved strength of ultrafine grain materials, increased strain hardening coefficient, and enhanced elongation to failure. Su et $\mathrm{al}^{26} \mathrm{com}-$ pared the strength of commercial-purity aluminum using ECAP at room temperature and that at cryogenic temperature with liquid nitrogen cooling. The cryogenic temperature ECAPed samples had higher hardness values than the room temperature ECAPed samples. The increased hardness of the cryogenic temperature ECAPed samples can be attributed to the existence of bulk mono and divacancies in these samples which are the major vacancy-type defects that can work as dislocations pinning centers and induce hardening.

A dislocation cell structure ${ }^{27}$ is assumed to form during deformation, which consist of dislocation cell wall $\left(\rho_{c}\right)$, statistical dislocation density $\left(\rho_{w s}\right)$ and geometrically necessary dislocation density $\left(\rho_{w g}\right)$. When the resolved shear strain rate $(\gamma)$ across the cell walls and cell interior are equal, $\rho_{c}, \rho_{w s}$, and $\rho_{w g}$ are governed by the following equations,

$\rho_{c}=\gamma\left(\alpha^{*} \frac{1}{\sqrt{3 b}} \sqrt{\rho_{w s}+\rho_{w g}}-\beta^{*} \frac{6}{b d(1-f)^{1 / 3}}-k_{0}\left(\frac{\gamma}{\gamma_{0}}\right)^{-1 / n} \rho_{c}\right)$

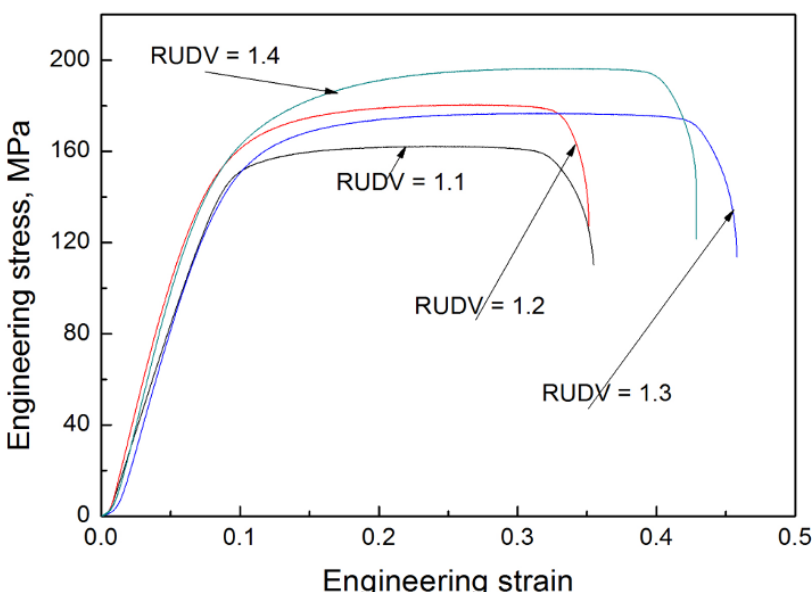

(a)

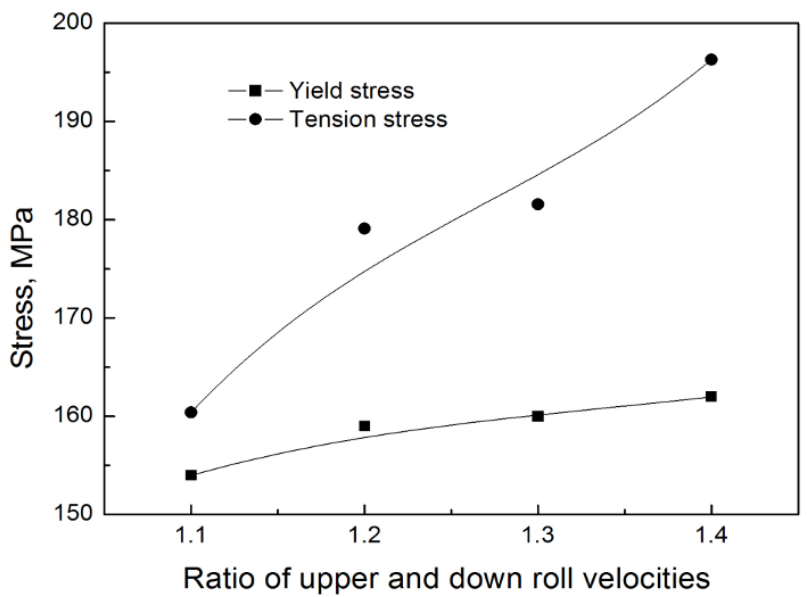

(b)

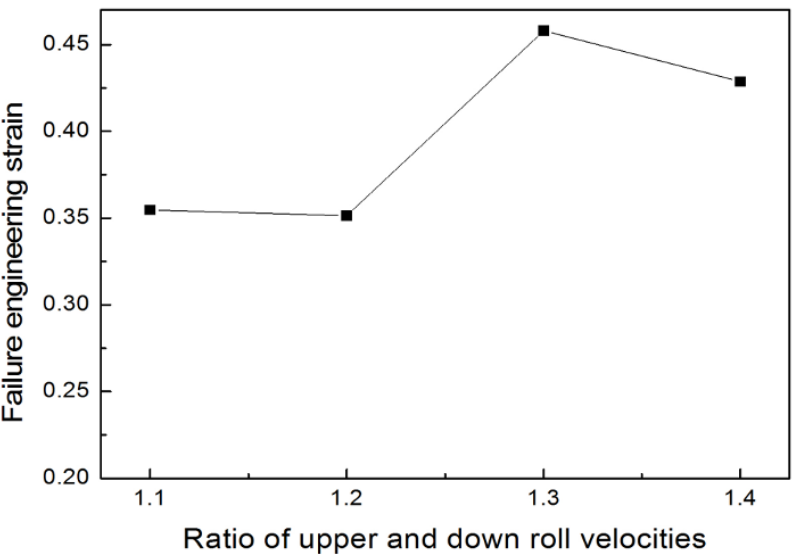

(c)

Figure $1 \mid$ Curve of engineering stress-strain (a), tensile and yield stress (b) and failure strain (c) of $\mathrm{Al} 1050$ under various ratios of upper and down rolling velocities.

$$
\begin{gathered}
\rho_{w s}=\gamma\left(\beta^{*} \frac{\sqrt{3}(1-f)}{f b} \sqrt{\rho_{w s}+\rho_{w g}}+(1-\xi) \beta^{*} \frac{6(1-f)^{2 / 3}}{b d f}-k_{0}\left(\frac{\gamma}{\gamma_{0}}\right)^{-1 / n} \rho_{w s}\right)(3) \\
\rho_{w g}=\gamma \xi \beta^{*} \frac{6(1-f)^{2 / 3}}{b d f}
\end{gathered}
$$

where $\alpha^{*}, \beta^{*}$ are dislocation evolution rate control parameters for the material; $n$ is a temperature sensitivity parameter, $n=\frac{B}{T}$, for pure 

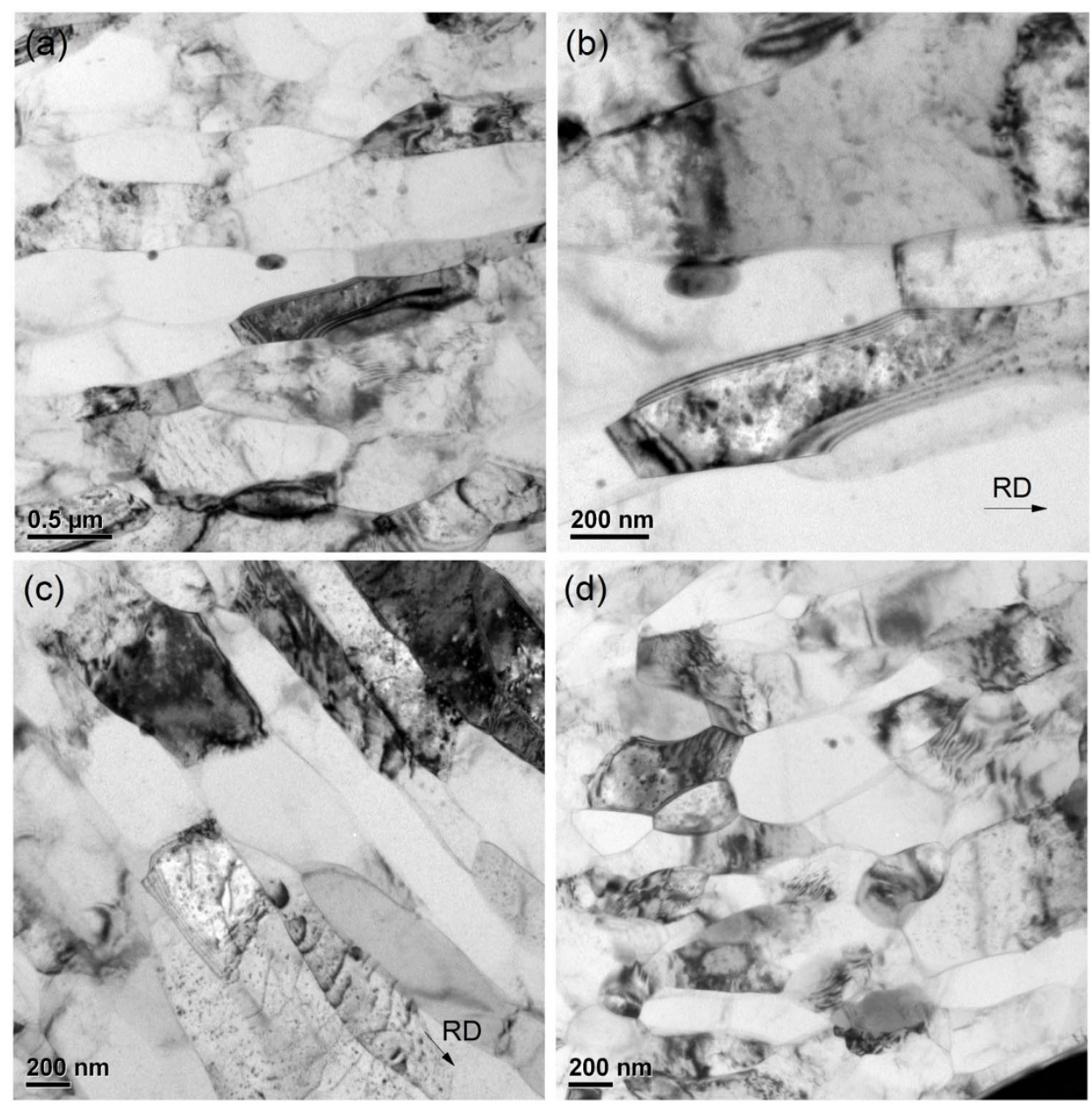

Figure 2 TEM micrograph of Al1050 after rolling with ratio of upper and down rolling velocities 1.1 for RD (a), (b) and 1.4 for RD(c) and TD(d) (RD rolling direction; TD - transverse direction).

aluminum, $\mathrm{B}=14900 \mathrm{~K}^{28}, T$ is temperature; $f$ is the volume fraction of the dislocation cell wall; $b$ is the magnitude of the Burgers vector of the material; $k_{0}$ is the dislocation annihilation rate parameter,

$$
k_{0}=1.334 \times 10^{-5} T^{2}-1.176 \times 10^{-3} T+6.14
$$

From the Eq (5), when the temperature is higher than the liquid nitrogen temperature, the dislocation density will increase with decreasing the temperature. Meanwhile, from the Eqs (2) (4), with increasing shear strain, the dislocation density will also increase.

Grain growth rate $(v)$, migration rate, and the driving force on the role of grain boundaries per unit area are on the following relation$\operatorname{ship}^{29}$ :

$$
v=m P
$$

where, $m$ is the grain boundary mobility, $P$ is the driving force of grain boundary movement, which can be related with the shear modulus $(\mu)$ and dislocation density $(\rho)$ of the material, as shown as follows,

$$
P=0.5 \rho \mu b^{2}
$$

From Eqs (6) and (7), with increasing the dislocation density, the grains of materials will be refined. It is reported that shear deformation plays a critical role in the grain refinement of materials processed by ECAPs as well as by asymmetric rolling ${ }^{30}$. The simple shear deformation through the thickness of the sheet is absolutely essential for property improvement, as well as the techniques ECAP, HPT, and asymmetric rolling. Very small recrystallized grains were observed in the adiabatic shear bands in metal when they were heavily deformed at high strain rates. Hines ${ }^{31}$ et al observed the recrystallized grains with 100-200 $\mathrm{nm}$ diameters within the shear bands of copper. Zuo et $\mathrm{al}^{32}$ observed the extremely fine grains with size of $500 \mathrm{~nm}$ in pure aluminum when asymmetric rolling process was used. With improved asymmetric rolling, the ability of grain refinement of asymmetric rolling is greatly improved.

This study, sheets are rolled between rolls that are rotating at different velocities in asymmetric cryorolling process. During asymmetric rolling, the complete strain state imposed to the strip is a combination of plane strain deformation and of an additional shear component imposed on the rolling plane in the rolling direction ${ }^{15}$. The asymmetric rolled strip exhibited uniform microstructures across the thickness direction, in contrast to the conventionally rolled strip, and a strength comparable to or exceeding that of the commercial $\mathrm{Al}$ alloy strip commonly in use. Fig. 3 shows the friction force distribution in the rolling deformation zone with increasing the RUDVs. In the normal rolling, the upper and down rolling velocities are the same, RUDV $=1.0$, there are mainly two zones that are forward zone and backward zone, as shown in Fig. 3(a). With the RUDV larger than 1.0, a shear zone appears in the deformation zone, as shown in Fig. 3(b). It is easy to understand that when the RUDV is larger than a certain value, the whole deformation zone will become shear zone, as shown in Fig. 3(c). During asymmetric rolling, the strain of sheet is combined by the plain strain and shear strain. With certain reduction ratio, the plain strain is certain. However, the shear strain in deformation zone will increase with increasing the RUDVs. 

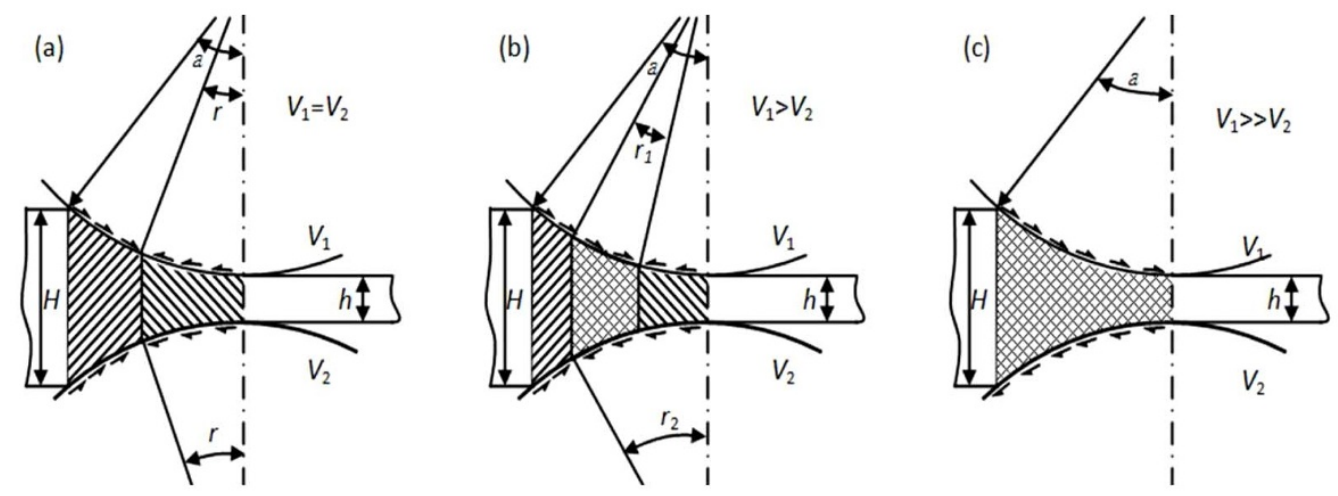

Figure 3 Friction force distribution in the deformation zone for normal rolling (a), asymmetric rolling for low RUDV (b), and asymmetric rolling for high RUDV (c).

For that, from Eqs (2) (4), the dislocation density increases with increasing the RUDVs.

A model has been developed for calculation of the uniform elongation of polycrystalline metals as a function of grain size by $\mathrm{Liu}^{33}$, as shown in Eq (8).

$$
\varepsilon_{u}=\varepsilon_{y}+\frac{\widehat{\sigma}_{s \infty}\left(1-\wedge_{s} / D\right)^{0.5}}{\theta_{0}} \ln \left(\frac{\hat{\sigma}_{s \infty}\left(1-\wedge_{s} / D\right)^{0.5}+\theta_{0}}{\hat{\sigma}_{s \infty}\left(1-\wedge_{s} / D\right)^{0.5}+\sigma_{y}}\right)
$$

where $\varepsilon_{u}$ is the uniform strain; the $\varepsilon_{y}$ is the elastic strain; $D$ is grain size; $\hat{\sigma}_{s \infty}$ is overall dislocation stress at steady state as $D=\infty ; \wedge_{s}$ is the dislocation cell size at steady state, it is a function of temperature and strain rate, but independent on strain and grain size. $\theta_{0}=C_{1} M^{2}{ }_{\alpha \mu / 2}$, $C_{1}$ is the probability for a moving dislocation of unit length to be stopped and subsequently stored at an obstacle, $M$ is Taylor orientation factor, $\alpha$ is a constant, $\mu$ is shear modulus; $\sigma_{y}$ is yield stress. In the model, it suggests that the occurrence of the instability of plasticity in ultrafine grained materials results from the lack of dislocation storages caused by the high density of high angle grain boundaries. In Fig. 2(a), there are many high angle grain boundaries when the RUDV is 1.1. However, the number of high angle grain boundaries is much less when the RUDV is 1.4, as shown in Fig. 2(c). Marker lines were scratched on the polished specimen surfaces using a nanoindenter to measure grain boundary sliding offsets during deformation by Liu and $\mathrm{Ma}^{34}$, which indicated that the grain boundary sliding contribution to the strain exceeded $50 \%$ and increased with increases in strain and temperature. Therefore, the high angle grain boundaries in the samples for RUDV of 1.1 should result in its lower ductility compared with that for RUDV of 1.4. Roumina et $\mathrm{al}^{15}$ also found that the $\mathrm{Al}$ alloy strips exhibit high formability when they are produced via the shear deformation.

The asymmetric cryorolling is first time used to produce nanostructural $\mathrm{Al} 1050$ sheets. When the ratio of upper and down rolling velocities is 1.4 , the $\mathrm{Al} 1050$ is of grain size with $211 \mathrm{~nm}$, which is much smaller than that obtain by traditional asymmetric rolling with $500 \mathrm{~nm}$. Both the strength and ductility of $\mathrm{Al} 1050$ materials increase with the ratio of upper and down rolling velocities from 1.1 to 1.4. When the ratio of upper and down rolling velocities is 1.4, the tensile stress reaches $196 \mathrm{MPa}$ which is larger by $22.3 \%$ than that for the ratio of upper and down rolling velocities of 1.1.

\section{Methods}

The commercial $1050 \mathrm{Al}$ alloy was used in this study. It was heat treated at $456^{\circ} \mathrm{C}$ for $1 \mathrm{~h}$ before rolling. Asymmetric cryorolling process was employed to produce $\mathrm{Al} 1050$ sheets with size of $1.45 \mathrm{~mm} \times 60 \mathrm{~mm} \times 200 \mathrm{~mm}$ under several of ratios of upper and down rolling velocities (RUDV). The RUDVs are 1.1, 1.2, 1.3, and 1.4 separately. Asymmetric cryorolling was performed by dipping the sheets into liquid nitrogen for at least $8 \mathrm{~min}$ before each rolling pass. The sheets were rolled to about $0.17 \mathrm{~mm}$ after seven rolling passes.
For the tensile tests, the rolled samples were machined into the ASTM subsized specimens with $25 \mathrm{~mm}$ gauge length. Uniaxial tensile tests were conducted with an initial strain rate of $1.0 \times 10^{-3} \mathrm{~s}^{-1}$ on an INSTRON machine operating at a constant crosshead speed.

Meanwhile, following asymmetric cryorolling, a FEI xT Nova Nanolab 200 Dualbeam workstation, which combines a focused ion beam and a field emission scanning electron microscope (FIB/SEM), was used to prepare thin-foil specimens from the $\mathrm{Al}$ sheets for further TEM observation. The electron beam was used to locate the region of interest on the sample surface, just like that in SEM. The FIB column forms an energetic beam of gallium ions, which scans over the sample surface for imaging as well as ion milling. This system is equipped with an in-situ platinum deposition system, which provides the localized protection on the top surface against the ion beam damage. The typical dimensions of TEM specimens are

$15 \mu \mathrm{m} \times 5 \mu \mathrm{m} \times 0.1 \mu \mathrm{m}$. During the FIB milling, an accelerating voltage of $30 \mathrm{kV}$ and high ion beam currents, e.g. $5 \mathrm{nA}$, were used for rough cutting, and lower beam currents of $1 \mathrm{nA}$ to $0.1 \mathrm{nA}$ were used for polishing both sides of the thin membrane. The electron transparent specimens were then placed on the standard carbon film $\mathrm{Cu}$ grid with the ex-situ lift-out method. A Philips CM200 field emission gun transmission electron microscope (FEGTEM) equipped with a Brucker energy dispersive $\mathrm{X}$-ray spectroscopy (EDAX) system was used to investigate the detailed microstructure operating at an accelerating voltage of $200 \mathrm{kV}$.

1. Loucif, A., Figueiredo, R. B., Baudin, T., Brisset, F. \& Langdon, T. G. Microstructural evolution in an Al-6061 alloy processed by high-pressure torsion. Mater. Sci. Eng. A 527, 4864-4869 (2010).

2. Guo, X., Remennik, S., Xu, C. \& Shechtman, D. Development of Mg-6.0\%Zn$1.0 \% \mathrm{Y}-0.6 \% \mathrm{Ce}-0.6 \% \mathrm{Zr}$ magnesium alloy and its microstructureal evolution during processing. Mater. Sci. Eng. A 473, 266-273 (2008).

3. Kim, J. K., Jeong, H. G., Hong, S. I., Kim, Y. S. \& Kim, W. I. Effect of aging treatment on heavily deformed microstructure of a 6061 aluminum alloy after equal channel angular pressing. Scripta Mater 45, 901-907 (2001).

4. Lee, S. H., Saito, Y., Sakai, T. \& Utsunomiya, H. Microstructures and mechanical properties of 6061 aluminum alloy processed by accumulative roll-bonding. Mater. Sci. Eng. A 325, 228-235 (2002).

5. Rezaei, M. R., Toroghinejad, M. R. \& Ashrafizadeh, F. Effects of ARB and ageing processes on mechanical properties and microstructure of 6061 aluminum alloy. J. Mater. Process. Technol 211, 1184-1190 (2011).

6. Pandey, S. C. et al. A theoretical and experimental evalution of repetitive corrugation and straightening, Application to Al-Cu and Al-Cu-Sc alloys. Mater. Sci. Eng. A 534, 282-287 (2012).

7. Lee, J. W. \& Park, J. J. Numerical and experimental investigations of constrained groove pressing and rolling for grain refinement. J. Mater. Process. Technol 130, 208-213 (2002)

8. Rusz, S. et al. SEM EBSD and TEM structure studies of a-brass after severe plastic deformation using equal channel rolling followed by groove pressing. Solid State Phenomena 186, 94-97 (2012).

9. Kawałek, A. The theoretical and experimental analysis of the effect of asymmetrical rolling on the value of unit pressure. J. Mater. Process. Technol 157-158, 531-535 (2004).

10. Kawalek, A., Dyja, H., Mroz, S. \& Knapinski, M. Effect of plate asymmetric rolling parameters on the change of the total unit pressure of roll. Metalurgija 50, 163-166 (2011).

11. Ji, Y. H. \& Park, J. J. Development of severe plastic deformation by various asymmetric rolling processes. Mater. Sci. Eng. A 499, 14-17 (2009).

12. Minh, T. N., Sidor, J., Petrov, R. \& Kestens, L. A. I. Texture evolution during asymmetrical warm rolling and subsequent annealing of electrical steel. Mater. Sci. Forum 702-703, 758-761 (2012).

13. Lee, J. K. \& Lee, D. N. Texture control and grain refinement of AA1050 Al alloy sheets by asymmetric rolling. Int. J. Mech. Sci 50(5), 869-887 (2008). 
14. Simoes, F., Sousa, R., Gracio, J., Barlat, F. \& Yoon, J. W. Effect of asymmetrical rolling and annealing the mechanical response of an 1050-O sheet. Int. J. Mater. Forming 2, 891-894 (2009).

15. Roumina, R. \& Sinclair, C. W. Deformation geometry and through-thickness strain gradients in asymmetric rolling. Metal. Mater. Trans. A 39, 2495-2503 (2008).

16. Zhao, Y. H., Liao, X. Z., Cheng, S., Ma, E. \& Zhu, Y. T. Simultaneously increasing the ductility and strength of nanostructured alloys. Adv. Mater 18, 2280-2283 (2006).

17. Wang, Y. M., Chen, M. W., Zhou, F. H. \& Ma, E. High tensile ductility in a nanostructured metal. Nature 419(31), 912-915 (2002).

18. Sushanta, K. P. \& Jayaganthan, R. Effect of ageing on microstructure and mechanical properties of bulk, cryorolled, and room temperature rolled $\mathrm{Al} 7075$ alloy. J. Alloy. Comp 509, 9609-9616 (2011).

19. Shanmugasundaram, T., Murty, B. S. \& Subramanya, S. V. Development of ultrafine grained high strength Al-Cu alloy by cryorolling. Scripta Mater 54, 2013-2017 (2006).

20. Rangaraju, N., Raghuram, T., Krishna, B. V., Prasad Rao, K. \& Venugopal, P. Effect of cryo-rolling and annealing on microstructure and properties of commercially pure aluminium. Mater. Sci. Eng. A 398, 246-251 (2005).

21. Zhu, Y. T. \& Liao, X. Z. Nanostructured metals, retaining ductility. Nature Mater 3, 351-352 (2004)

22. Nageswara rao, P. \& Jayaganthan, R. Effects of warm rolling and ageing after cryogenic rolling on mechanical properties and microstructure of $\mathrm{Al} 6061$ alloy. Mater. Des 39, 226-233 (2012).

23. Sato, Y. S., Urata, M., Kokawa, H. \& Ikeda, K. Hall-Petch relationship in friction stir welds of equal channel angular-pressed aluminium alloys. Mater. Sci. Eng. A 354, 298-305 (2003)

24. Gang, U. G., Lee, S. H. \& Nam, W. J. The evolution of microstructure and mechanical properties of a 5052 aluminium alloy by the application of cryogenic rolling and warm rolling. Mater. Trans 50, 82-86 (2009).

25. Moreno-Valle, E. C., Sabirov, I., Perez-Prado, M. T., Yu Murashkin, M., Bobruk, E. V. \& Valiev, R. Z. Effect of the grain refinement via severe plastic deformation on strength properties and deformation behavior of an Al6061 alloy at room and cryogenic temperatures. Mater. Let 65, 2917-2919 (2011).

26. Su, L. H. et al. Study of vacancy-type defects by positron annihilation in ultrafinegrained aluminum severely deformed at room and cryogenic temperatures. Acta Mater 60, 4218-4228 (2012).

27. Ding, H., Shen, N. \& Shin, Y. Predictive modeling of grain refinement during multi-pass cold rolling. J. Mater. Process. Technol 212, 1003-1013 (2012).

28. Baik, S. C., Estrin, Y., Kim, H. S. \& Hellming R. J. Dislocation density-based modeling of deformation behavior of aluminum under equal channel angular pressing. Mater. Sci. Eng. A 351, 86-97 (2003).
29. Hallberg, H., Wallin, M. \& Ristinmaa, M. Simulation of discontinuous dynamic recrystallizaiton in pure $\mathrm{Cu}$ using a probalilistic cellular automaton. Comp. Mater. Sci 49, 25-34 (2010)

30. Kim, W. J., Lee, J. B., Kim, W. Y., Jeong, H. T. \& Jeong, H. G. Microstructure and mechanical properties of $\mathrm{Mg}-\mathrm{Al}-\mathrm{Zn}$ sheets severely deformed by asymmetrical rolling. Scripta Mater 56, 309-312 (2007).

31. Hines, J. A. \& Vecchio, K. S. Recrystallization kinetics with adiabatic shear bands Acta Mater 45, 635-649 (1997).

32. Zuo, F. Q., Jiang, J. H., Shan, A. D., Fang, J. M. \& Zhang, X. Y. Shear deformation and grain refinement in pure $\mathrm{Al}$ by asymmetric rolling. T. Nonfer. Metal. Soc. China 18, 774-777 (2008).

33. Liu, W. J. Modelling the dependence of uniform elongation on grain size. Mater. Sci. Forum 539-543, 2576-2581 (2007).

34. Liu, F. C. \& Ma, Z. Y. Contribution of grain boundary sliding in low-temperature superplasticity of ultrafine-grained aluminum alloys. Scripta Mater 62, 125-128 (2010).

\section{Acknowledgements}

The authors gratefully acknowledge the financial supports from the National Natural Science Foundation of China through 51105071, the Doctorate Foundation of the Ministry of Education of China through the Grant. 20090042120005, and the Vice-Chancellor's Fellowship Grant at University of Wollongong. The authors also want to thanks Dr. Hongyan WU, Xiangkun SUN, Delin TANG, in Northeastern University, Shenyang.

\section{Author contributions}

YH conceived the study. YH, LC, TK, LX and KC wrote the main manuscript text. YH, LX, SY and KC conducted the experiments. YH, LC, TK, LX, YQ analyzed the data. All authors reviewed the manuscript.

\section{Additional information}

Competing financial interests: The authors declare no competing financial interests.

License: This work is licensed under a Creative Commons Attribution-NonCommercial-ShareAlike 3.0 Unported License. To view a copy of this license, visit http://creativecommons.org/licenses/by-nc-sa/3.0/

How to cite this article: YU, H. et al. Asymmetric cryorolling for fabrication of nanostructural aluminum sheets. Sci. Rep. 2, 772; DOI:10.1038/srep00772 (2012). 